\title{
Uma Revisão Sistemática de Instrumentos sobre Qualidade de Vida em Pessoas Idosas após Implante Coclear
}

\author{
Maria Piedade Brandão ${ }^{a, b} \quad$ Cristiana Rebeloc \\ ${ }^{a}$ Escola Superior de Saúde da Universidade de Aveiro, Aveiro, Portugal; ${ }^{b}$ CINTESIS-UA - Centro de Investigação \\ em Tecnologias e Serviços de Saúde, Universidade de Aveiro/Universidade do Porto, Porto, Portugal; \\ 'Secção Autónoma das Ciências da Saúde, Universidade de Aveiro, Aveiro, Portugal
}

\section{Palavras Chave}

Qualidade de vida $\cdot$ Idoso $\cdot$ Deficiência auditiva $\cdot$ Implante coclear

\section{Resumo}

Introdução: A deficiência auditiva é um problema particular de saúde que afeta mais de 275 milhões de pessoas em todo o mundo e é o défice sensorial mais comum nas pessoas idosas. O objetivo deste estudo foi identificar os instrumentos para avaliar a qualidade de vida em pessoas idosas com deficiência auditiva submetidas ao implante coclear e rever os estudos que incluem a avaliação da qualidade de vida na sua relação com a especificidade da deficiência auditiva pós-lingual sensorioneural severa a profunda bilateral nessa população. Métodos: Foi conduzida uma revisão sistemática da literatura entre Janeiro de 1900 e Junho de 2012. Instrumentos validados e usados em pessoas com 65 anos de idade ou mais, com deficiência auditiva pós-lingual, sensorioneural severa a profunda, que tivessem sido sujeitas a implante co-

\section{KARGER}

E-Mail karger@karger.com www.karger.com/pjp (c) 2017 The Author(s). Published by S. Karger AG, Basel on behalf of Escola Nacional de Saúde Pública Karcer 0 pen eccess

This article is licensed under the Creative Commons AttributionNonCommercial-NoDerivatives 4.0 International License (CC BYNC-ND) (http://www.karger.com/Services/OpenAccessLicense) $\mathrm{NC}-\mathrm{ND})$ (http://www.karger.com/Services/OpenAccessLicense).
Usage and distribution for commercial purposes as well as any distribution of modified material requires written permission. clear, foram revistos. A pesquisa bibliográfica foi limitada a estudos escritos em língua inglesa. Resultados: A revisão identificou um total de sete instrumentos validados. Destes, um foi especialmente desenhado para avaliar o impacto do implante coclear e seis para as condições gerais das pessoas com deficiência auditiva. A qualidade metodológica de todos os artigos foi avaliada segundo as recomendações de STROBE e todos os artigos foram classificados na categoria B. Conclusões: Os resultados desta revisão sistemática podem ajudar os profissionais de saúde e os investigadores a selecionarem os instrumentos mais apropriados para avaliar as mudanças na qualidade de vida dos idosos com implantes cocleares.

(C) 2017 The Author(s). Published by S. Karger AG, Basel on behalf of Escola Nacional de Saúde Pública

This article was supported by ERDF through the operation POCI01-0145-FEDER-007746 funded by the Programa Operacional Competitividade e Internacionalização - COMPETE2020 and by National Funds through FCT - Fundação para a Ciência e a Tecnologia within CINTESIS, R\&D Unit (reference UID/IC/4255/2013).
Maria Piedade Brandão

Escola Superior de Saúde da Universidade de Aveiro Edifício 30, Agras do Crasto, Campus Universitário de Santiago PT-3810-193 Aveiro (Portugal)

E-Mail mpiedade@ua.pt 
A Systematic Review of Quality of Life Instruments in the Elderly after Cochlear Implantation

\author{
Keywords \\ Quality of life $\cdot$ Elderly $\cdot$ Hearing loss $\cdot$ Cochlear \\ implantation
}

\begin{abstract}
Introduction: Hearing loss is a health problem that affects over 275 million people worldwide and it is the most common sensory deficit in the elderly. The aim of this study was to identify quality of life instruments that have been validated in older people with hearing loss submitted to cochlear implant and to review the studies that have used the quality of life instruments in their relationship with the specificity of postlingual bilateral profound sensorineural hearing loss in this population. Methods: A systematic literature search was conducted from January 1900 to June 2012. Validated instruments that were used in persons aged $\geq 65$ years with postlingual bilateral profound sensorineural hearing loss who underwent cochlear implantation were reviewed. The search was limited to studies in English language. Results: The review identified a total of 7 validated instruments. Of these, 1 was specially designed for the specific disease and 6 for the general conditions of persons with hearing loss. The methodological quality of all articles was assessed according to the STROBE recommendations, and all items were classified in category B. Conclusions: The results of this systematic review can help health professionals and researchers select the most appropriate instruments to assess the changes in quality of life in elderly with cochlear implantation.

(c) 2017 The Author(s). Published by S. Karger AG, Basel on behalf of Escola Nacional de Saúde Pública
\end{abstract}

\section{Introdução}

A deficiência auditiva (DA) afeta milhões de pessoas em todo o mundo [1], tendo sido considerada como uma das doenças crónicas mais frequentes na população idosa $[2,3]$. A presbiacusia é a perda de acuidade auditiva relacionada com o envelhecimento, sendo que é a causa mais comum de DA sensorioneural nas pessoas idosas [4]. Vários estudos têm mostrado que a DA sensorioneural severa a profunda bilateral, provoca frequentemente alterações na qualidade de vida (QDV) dessas pessoas [5].

Instrumentos sobre QDV em Pessoas

Idosas após Implante Coclear
Pode-se observar na literatura que a DA interfere em diferentes domínios da QDV: emocional, social, psicológico e intelectual $[2,6,7]$. Assim, têm sido descritas as implicações na solidão, depressão, ansiedade e declínio cognitivo [7-10].

O implante coclear tem sido a opção mais viável para a reabilitação auditiva da DA sensorioneural severa a profunda bilateral [11]. Os benefícios auditivos e psicossociais decorrentes da aplicação do implante coclear têm sido extensivamente documentados, assim como a QDV associada a essa técnica de reabilitação em pessoas de várias faixas etárias incluindo as pessoas idosas $[11,12]$. Para os clínicos e investigadores, é importante monitorizar, avaliar e comparar os efeitos do implante coclear na QDV das pessoas idosas, assim como monitorizar o efeito das melhorias técnicas do implante coclear [13].

Apesar de existir uma grande diversidade de instrumentos de avaliação da QDV, os mesmos integram conteúdos muito genéricos e, embora direcionados a qualquer idade, na sua globalidade não se debruçam sobre problemas específicos nomeadamente em relação à DA. O objetivo desta revisão sistemática foi: (1) identificar os instrumentos disponíveis para avaliar a QDV em pessoas idosas com DA submetidas ao implante coclear; e (2) rever os estudos que incluem a avaliação da QDV na sua relação com a especificidade da DA pós-lingual sensorioneural severa a profunda bilateral nessa população.

\section{Métodos}

Estratégia de Pesquisa

Tendo em conta os princípios orientadores previstos em Preferred Reporting Items for Systematic Reviews and Meta-Analyses (PRISMA), os estudos foram procurados em bases de dados eletrónicas, publicadas entre Janeiro de 1900 e Junho de 2012 (Tabela 1) designadamente na Academic Search Complete, Medline with Full Text, PubMed, Science Direct, Scopus, Web of Knowledge, Web of Science e Wiley Online Library. As estratégias de pesquisa basearam-se no uso duma combinação de palavras, que deveriam ser encontradas no título, resumo ou palavras-chave do artigo: cochlear implant, elderly, old people, old adult, senior, geriatric, quality of life, postlingual, deaf, hearing loss, hearing impairment. Às palavras-chave foram adicionados operadores booleanos (AND e OR) e um símbolo de truncatura $\left(^{*}\right)$ para alargar ou restringir a pesquisa. Os dados foram extraídos por um revisor (CR) e verificada a exatidão e integridade por um segundo revisor que agiu como árbitro (MPB). As divergências foram resolvidas por meio de discussão com os peritos da área. Na Figura 1 encontra-se um diagrama que descreve todo o processo de seleção dos estudos. $\mathrm{O}$ acesso à versão integral de artigos foi conseguido através da Virtual Private Network, disponibilizada pela Universidade de Aveiro.

Identificaram-se dois tipos de instrumentos de avaliação da QDV: (i) genéricos e (ii) específicos (área da otorrinolaringologia). 
Table 1. Bases de dados eletrónicas utilizadas no estudo

\begin{tabular}{ll}
\hline Bases de dados & Fonte/ano \\
\hline Academic Search Complete & EBSCO/1950-2012 (até Setembro) \\
Medline with Full Text & EBSCO/1965-2012 (até Setembro) \\
PubMed & Elsevier/1950-2012 (até Setembro) \\
Science Direct & Elsevier/1950-2012 (até Setembro) \\
Scopus & Elsevier/1996-2012 (até Setembro) \\
Web of Knowledge & ISI/1900-2012 (até Setembro) \\
Web of Science & ISI/1900-2012 (até Setembro) \\
Wiley Online Library & $1950-2012$ (até Setembro)
\end{tabular}

Fig. 1. Apresentação esquemática da metodologia usada nos critérios de seleção baseada diagrama de fluxo QUOROM (Quality Of Reporting Of Meta-Analyses).

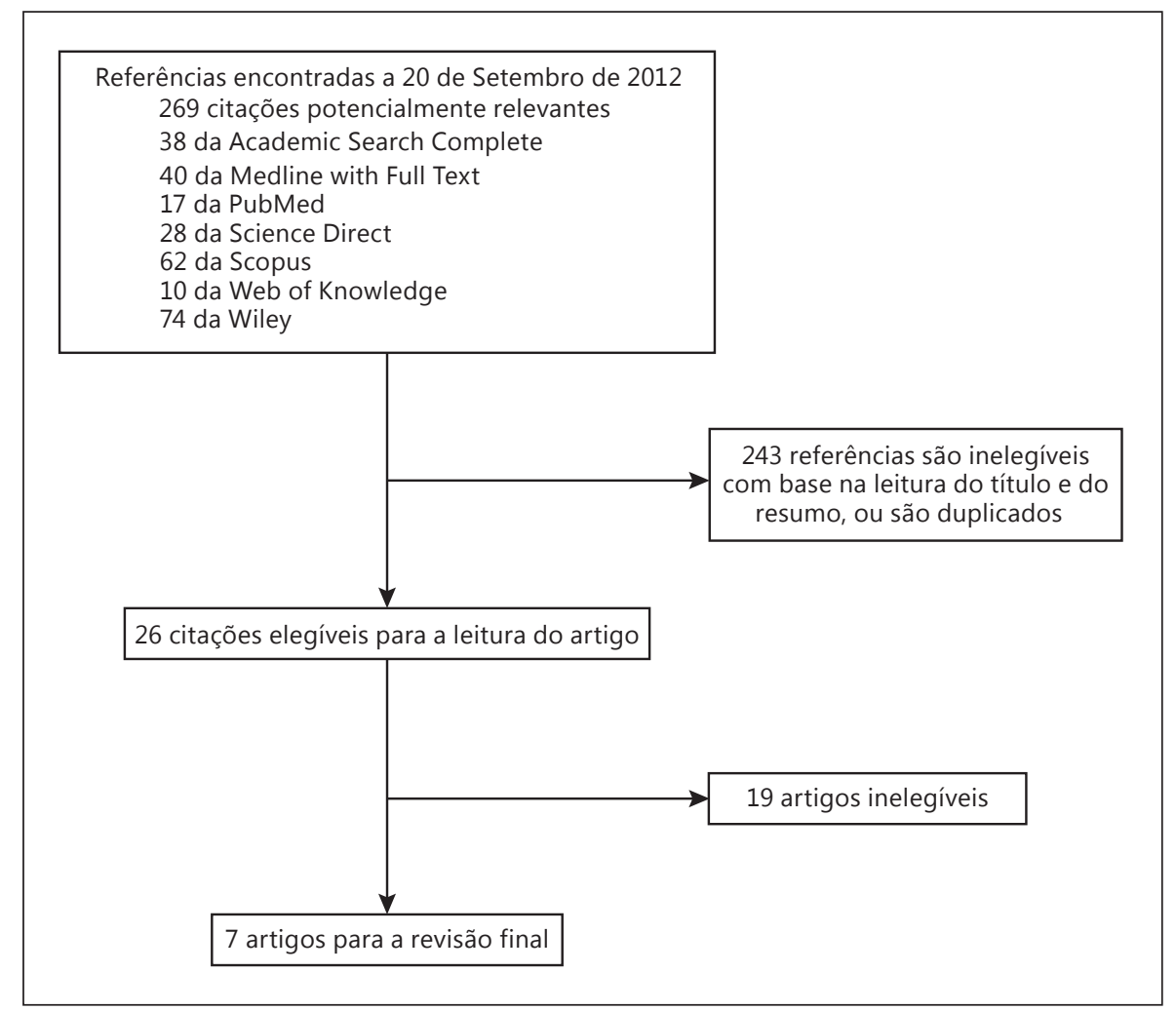

São designados por instrumentos de avaliação da QDV genéricos, aqueles que podem ser aplicados a qualquer indivíduo, doente ou saudável e que aborda geralmente três dimensões: física, mental/ psicológica e social. Os instrumentos de avaliação da QDV específicos medem a QDV e as suas alterações em situações específicas da área da otorrinolaringologia, nomeadamente na DA e no implante coclear. Para identificar o tipo dos instrumentos de QDV, foram extraídas as informações relativas aos domínios (físico, psicológico, dor física, saúde em geral, vitalidade, função social e saúde mental) e pontuações. Foi também extraída informação acerca da validação dos instrumentos de avaliação da QDV.

\section{Critérios de Seleção dos Estudos}

Para a inclusão dos estudos, foram considerados os seguintes critérios: (i) artigos de investigação originais e escritos em língua inglesa; (ii) participantes idosos com DA pós-lingual, sensorioneural, severa a profunda, e que tenham sido implantados com 65 anos de idade ou mais; (iii) aplicação de um instrumento de avaliação da QDV das pessoas idosas sobre um ou mais domínios: físico, psicológico, dor física, saúde em geral, vitalidade, função social e saúde mental; iv) com instrumentos validados.

Foram excluídos os estudos cujos instrumentos utilizados na avaliação da QDV tinham em conta somente as preferências das pessoas.

\section{Qualidade Metodológica dos Estudos}

A qualidade metodológica dos estudos incluídos nesta revisão foi avaliada segundo as recomendações de Strengthening the Reporting of Observational Studies in Epidemiology (STROBE) [14, $15]$. 
Table 2. Descrição geral dos estudos selecionados para a revisão sistemática

\begin{tabular}{|c|c|c|c|}
\hline Autores & Tipo de estudo & $n$; idade de implantação & Métodos de avaliação da qualidade de vida \\
\hline Vermeire et al. [23] & Transversal & $\begin{array}{l}\text { Grupo } 1: n=33 ;<55 \text { anos } \\
\text { Grupo 2: } n=31 ; 56-69 \text { anos } \\
\text { Grupo 3: } n=25 ; \geq 70 \text { anos }\end{array}$ & $\begin{array}{l}\text { Hearing Handicap Inventory for the Adults (HHIA); } \\
\text { Glasgow Benefit Inventory Questionnaire (GBI) }\end{array}$ \\
\hline Orabi et al. [20] & Retrospetivo & $n=34 ; 65-80$ anos & $\begin{array}{l}\text { Glasgow Health Status Inventory Questionnaire (GHSI); } \\
\text { Glasgow Benefit Inventory Questionnaire (GBI) }\end{array}$ \\
\hline Eshraghi et al. [18] & Retrospetivo & $n=21 ; \geq 79$ anos & Hearing Handicap Inventory for the Elderly (HHIE) \\
\hline Park et al. [21] & Retrospetivo & $\begin{array}{l}\text { Grupo } 1: n=61 ;<50 \text { anos } \\
\text { Grupo 2: } n=50 ; 51-65 \text { anos } \\
\text { Grupo 3: } n=50 ;>65 \text { anos }\end{array}$ & $\begin{array}{l}\text { Hearing Handicap Inventory for the Adults (HHIA); } \\
\text { Hearing Handicap Inventory for the Elderly (HHIE) }\end{array}$ \\
\hline Chung et al. [17] & Prospetivo & $\begin{array}{l}\text { Grupo } 1: n=92 ;<45 \text { anos } \\
\text { Grupo 2: } n=123 ; 45-65 \text { anos } \\
\text { Grupo 3: } n=68 ;>65 \text { anos }\end{array}$ & $\begin{array}{l}\text { Medical Outcome Study } 36 \text { Short Form Healthy Survey } \\
\text { (SF-36) }\end{array}$ \\
\hline
\end{tabular}

Tendo por base outros autores [16], foram consideradas três categorias na avaliação: A - quando o estudo preenchia mais de $80 \%$ dos critérios estabelecidos nas recomendações STROBE; B quando $50-80 \%$ dos critérios do STROBE fossem preenchidos; C - quando menos de 50\% dos critérios fossem preenchidos.

\section{Resultados}

\section{Descrição dos Estudos}

Após o processo de localização e seleção dos artigos detalhado na Figura 1, preencheram os critérios de elegibilidade sete estudos [17-23]. Em todos os estudos verificou-se que o impacto da colocação dos implantes cocleares na QDV das pessoas idosas era positivo, isto é, houve melhoria na QDV após essa técnica de intervenção.

A Tabela 2 reúne as informações de cada artigo selecionado para esta revisão acerca do tipo de estudo, do número de participantes e respetiva idade aquando da colocação do implante coclear e resume ainda os instrumentos de avaliação de QDV usados.

Os instrumentos Glasgow Health Status Inventory Questionnaire (GHSI) e Glasgow Benefit Inventory Questionnaire (GBI) desenhados respetivamente para medir o efeito de um problema de saúde sobre a QDV de uma pessoa e a QDV após as intervenções cirúrgicas, foram usados em dois estudos: Vermeire et al. [23] e Orabi et al. [20]. O instrumento Hearing Handicap Inventory for the Elderly (HHIE) ou Adults (HHIA) desenhado para avaliar a perceção da QDV de pessoas com DA com 65 anos ou mais (HHIE) e abaixo dos 65 anos (HHIA), tem um enfoque especial para as consequências emocionais e sociais percecionadas em função da perda de audição e foi utilizado em três estudos: Vermeire et al. [23], Eshraghi et al. [18] e Park et al. [21].

O instrumento SF-36 de cariz genérico desenhado para medir os domínios físico e psicológico da QDV foi utilizado em dois estudos: Chung et al. [17] e Olze et al. [19].

Os principais resultados e conclusões de cada estudo estão resumidos na Tabela 3. Nos estudos de Vermeire et al. [23], Poissant et al. [22] e Park et al. [21] os participantes idosos apresentaram melhorias significativas na QDV após o implante coclear, semelhantes àquelas que são apresentadas por indivíduos adultos com menos idade. No estudo de Olze et al. [19] as pessoas idosas em comparação com o grupo de pessoas mais novas, revelaram melhorias significativas na QDV após o implante coclear. No estudo de Chung et al. [17], os participantes idosos 
Table 3. Melhorias na QDV após o implante coclear

\begin{tabular}{|c|c|c|c|}
\hline Autores & Participantes, $n$ & Instrumento & Melhorias significativas na QDV e saúde após o implante coclear \\
\hline $\begin{array}{l}\text { Vermeire et al. } \\
{[23]}\end{array}$ & 89 & HHI & $\begin{array}{l}\text { Nos domínios situacional e emocional (subescala situacional e emocional) nos } \\
\text { participantes entre os } 56 \text { e os } 69 \text { anos; no domínio situacional (subescala } \\
\text { situacional) nos participantes com } \geq 70 \text { anos } \\
\text { Os participantes com < } 55 \text { anos mostraram benefícios significativos nas } 3 \\
\text { subescalas (geral, social e física) }\end{array}$ \\
\hline Poissant et al. [22] & 26 & $\begin{array}{l}\text { GDS } \\
\text { UCLA }\end{array}$ & $\begin{array}{l}\text { Diminuição da depressão } \\
\text { Diminuição do grau de solidão }\end{array}$ \\
\hline Eshraghi et al. [18] & 21 & HHI-E & $\begin{array}{l}\text { Diminuição das dificuldades apresentadas, em todos os domínios (subescala } \\
\text { situacional e emocional), quando o implante coclear se encontra ligado }\end{array}$ \\
\hline Chung et al. [17] & 283 & SF-36 & $\begin{array}{l}\text { Em } 5 \text { domínios (desempenho físico e emocional, vitalidade, função social, } \\
\text { saúde mental) nos participantes com }<45 \text { anos e entre os } 45 \text { e os } 65 \text { anos e em } \\
2 \text { domínios (função social, saúde mental) nos participantes com mais de } 65 \\
\text { anos }\end{array}$ \\
\hline
\end{tabular}

apresentaram melhorias significativas na QDV em comparação com os mais novos, contudo as pessoas com menos idade mostraram melhorias mais acentuadas em alguns domínios da QDV (desempenho físico e emocional, vitalidade, função social e saúde mental).

Nos estudos de Orabi et al. [20] e Eshraghi et al. [18], os participantes apresentaram melhorias significativas em todos os domínios da QDV avaliados, após a colocação do implante coclear.

\section{Instrumentos de Avaliação}

Instrumentos de Avaliação de QDV Genéricos

Tendo em conta os critérios de inclusão pré-definidos, não foram encontrados outros instrumentos genéricos além dos seguintes: Glasgow Benefit Inventory (GBI) e o Glasgow Health Status Inventory (GHSI). O GBI e o GHSI foram empregues nos estudos de Vermeire et al. [23] e Orabi et al. [20]. O GBI é um questionário genérico que mede as alterações na QDV (perceção geral de bem-estar psicológico, social e físico) produzidas pelas intervenções cirúrgicas [24]. O GHSI mede o efeito de um problema de saúde na QDV de uma pessoa. Este permite a comparação entre várias condições de saúde, diferentes intervenções de saúde e entre subgrupos demográficos e culturais. Ambos os questionários contêm 18 questões, as quais podem ser completadas através de uma entrevista ou pelo próprio paciente.

Medical Outcome Study 36 Short Form Healthy Survey (MOS SF-36). O SF-36 [25, 26] é considerado uma medida genérica de saúde, uma vez que se destina a medir conceitos de saúde que representam valores humanos básicos relevantes à funcionalidade e ao bem-estar de cada um, sendo que não é específico de qualquer nível etário, doença ou tratamento [25]. Este questionário foi empregue nos estudos de Olze et al. [18] e Chung et al. [17]. O SF-36 foi adaptado linguística e culturalmente e validado para o português [25, 27]. Esta escala foi construída para representar oito dos conceitos mais importantes de saúde: função física, desempenho físico e emocional, dor física, saúde em geral, vitalidade, função social e saúde mental. Estas oito escalas podem ser agrupadas em duas componentes: saúde física e saúde mental [25].

Geriatric Depression Screening Scale (GDS). A GDS [28] é um questionário de 30 itens, desenvolvido e valida-
Brandão/Rebelo 
do para avaliar a depressão nas pessoas idosas, independentemente do seu estado de saúde. Este, foi empregue no estudo de Poissant et al. [22] e tem sido considerado por alguns autores como fazendo parte do grupo de instrumentos genéricos de medida da qualidade de vida [29]. Os indivíduos têm de responder "sim" ou "não" a cada questão, tendo em consideração aquilo que sentiram na semana anterior, sendo que 20 questões indicam a presença de depressão quando a resposta é "sim", enquanto as restantes 10 questões indicam a presença de depressão quando a resposta é "não" [28]. O estudo realizado por Poissant [22], mostra que as pessoas idosas participantes tinham graus mais elevados de solidão e depressão antes dos implantes cocleares e por isso uma pior QDV devido à dificuldade em comunicar.

UCLA Loneliness Scale (version 3). A UCLA Loneliness Scale (version 3) [30] é considerada uma medida fiável para avaliar a solidão nas pessoas idosas, composta por 20 questões que descrevem como as pessoas se sentem sobre si mesmas, as pessoas à sua volta e os seus relacionamentos. Esta escala foi empregue no estudo de Poissant et al. [22].

Os indivíduos indicam os seus sentimentos respondendo a questões numa escala de 4 pontos $(1=$ nunca a 4 = sempre). Existem 11 itens redigidos de forma negativa e 9 itens positivos [30]. As pontuações mais elevadas indicam um grau de solidão maior. No que respeita à solidão, a evidência científica tem mostrado que as pessoas idosas muitas vezes expressam sentimentos negativos e de solidão que resultam de uma combinação do processo de envelhecimento e de estereótipos sociais, influenciando a respetiva qualidade de vida [31]. Por esta razão, a solidão pode ser um indicador crítico para estimar a qualidade de vida assim como vice-versa [32] tendo sido este o motivo que levou a incluir a a UCLA nesta revisão.

Instrumentos de Avaliação de QDV Específicos

Nijmegen Cochlear Implant Questionnaire (NCIQ). O Nijmegen Cochlear Implant Questionnaire (NCIQ) foi desenvolvido e validado [13] com o objetivo de avaliar a QDV dos indivíduos utilizadores de implante coclear, tendo sido utilizado no estudo de Olze et al. [18] Este questionário avalia três domínios principais, especificados em seis subdomínios. O domínio físico engloba três subdomínios, relacionados com a perceção de sons (básica e avançada) e a produção de fala. O domínio psicológico avalia a autoestima e, finalmente, do domínio social fazem parte os subdomínios relacionados com a atividade e as interações sociais. Cada subdomínio tem associado 10 itens, que correspondem a 10 questões, pelo que no total o questionário é composto por 60 perguntas. Para cada questão é apresentada uma escala de resposta com 5 opções, que varia de "nunca" a "sempre" (em 55 perguntas) ou de "não" a "bom" (nas restantes 5 perguntas). Caso determinada pergunta não se aplique ao indivíduo, pode ser dada uma sexta resposta: "não aplicável". Desde a sua validação, o NCIQ tem sido utilizado como um teste padrão para a avaliação da QDV dos indivíduos com implante coclear ou prótese auditiva. Em apenas dois dos seis subdomínios (produção de fala e autoestima), a consistência interna mostrou-se aceitável (0,73 e 0,75 respetivamente) enquanto os restantes quatro subdomínios (perceção de sons básica e avançada, atividade e interações sociais), apresentam uma consistência interna alta $(>80)$ [13]. No que respeita à fiabilidade teste-reteste, os mesmos autores mostram resultados satisfatórios para o instrumento NCIQ.

Hearing Handicap Inventory (HHI). O HHI é um instrumento de autoavaliação desenvolvido com o objetivo de avaliar os efeitos da DA no ajustamento social e emocional das pessoas idosas, em que grau os indivíduos se sentem restringidos ou em desvantagem nos ambientes sociais e pessoais [33]. Este instrumento foi empregue nos estudos de Vermeire et al. [23], Eshraghi et al. [18] e Park et al. [21]. O HHI é composto por duas subescalas: uma subescala de 13 itens que explora as consequências emocionais da DA; uma subescala de 12 itens que aborda os efeitos sociais e situacionais. Cada item tem três possibilidades de resposta: "sim", "por vezes" e "não", sendo que são atribuídos 4 pontos a uma resposta "sim", dois pontos ao "por vezes" e zero pontos a uma resposta "não", pelo que poderão, no máximo, ser obtidos 100 pontos $[33,34]$. As pontuações de HHI mais elevadas indicam um maior grau de incapacidade/dificuldades [33, 34]. Existem duas variações similares do HHI, o Hearing Handicap Inventory for the Adults (HHIA) [34] e o Hearing Handicap Inventory for the Elderly (HHIE) [33]. O HHIE foi desenvolvido e validado para ser usado em pessoas com 66 anos ou mais de idade. O HHIA foi criado, através da modificação do HHIE, para ser usado em indivíduos com DA que tenham menos de 65 anos [34]. Os critérios utilizados pelos vários autores selecionados nesta revisão são diferentes, nomeadamente: (i) a definição da idade cronológica, a partir da qual consideram os participantes como pessoas idosas (65 anos de idade ou mais e acima dos 70 anos); e (ii) a definição do intervalo de tempo considerado para a avaliação dos pacientes após a implantação, para que sejam comparados os desempenhos pré e pósimplante coclear (1 ano e 2 anos). 
Qualidade Metodológica dos Estudos

Através da aplicação dos critérios STROBE, todos os estudos têm uma alta credibilidade pois concentraram-se na categoria B pré-definida. Não existem grandes diferenças de qualidade entre os estudos, tendo em conta as percentagens obtidas por cada um: todos os estudos cumpriram entre $50-80 \%$ dos critérios de STROBE. O estudo que obteve uma percentagem mais elevada $(77,2 \%)$ segundo os critérios STROBE, foi o de Chung et al. [17]. Mediante os mesmos critérios, a percentagem mais baixa $(68,2 \%)$ foi observada em quatro estudos [18, 21-23].

\section{Discussão}

Os objetivos desta revisão da literatura foram identificar e avaliar os instrumentos disponíveis que avaliam a QDV em pessoas idosas com DA, pós-lingual sensorioneural severa a profunda bilateral, com implante coclear. Nos estudos identificados nesta revisão, foram encontrados sete instrumentos usados na avaliação da QDV das pessoas idosas.

Apenas um dos instrumentos de avaliação foi especialmente desenhado para a avaliação da QDV das pessoas com implante coclear [19], designado por NCIQ, sendo que este foi o primeiro instrumento criado especificamente para a avaliação das pessoas com implante coclear [13].

O instrumento HHI foi utilizado em três estudos [18, $21,23]$ para avaliar a QDV a pessoas com DA sem a especificidade de a particularizar. Os instrumentos GBI, GHSI, SF-36, GDS e a UCLA Loneliness Scale (version 3) utilizados em 5 estudos $[16,18-22]$ embora também avaliem a QDV, não foram especificamente desenhados para uma determinada área ou patologia podendo ser encontrados em diversos estudos, que abordam diferentes temáticas.

A colocação do implante coclear na população idosa tem sido bastante debatida, dada a possibilidade destes indivíduos poderem não obter tantos benefícios com a colocação do implante coclear quanto as pessoas mais jovens, devido a fatores audiológicos (alterações nos sistemas auditivos relacionados com a idade), físicos e cognitivos $[23,35]$.

Contudo, os estudos analisados são unânimes em considerar que as pessoas idosas têm benefícios na QDV após a colocação do implante coclear. Após a análise de todos os estudos, verificou-se que existem estudos nos quais não são observadas diferenças estatisticamente significativas entre o desempenho das pessoas idosas e dos adultos mais jovens, sendo que outros estudos referem a existên- cia de menos ou mais ganhos significativos nas pessoas idosas.

Estudos de Olze et al. [19], Poissant et al. [22] e Vermeire et al. [23], indicam que os participantes idosos com idade igual ou superior a 70 anos com implante coclear apresentaram melhorias significativas na QDV. Contudo, essas melhorias foram semelhantes às dos pacientes adultos com menos idade, dado que foi feita a comparação dos resultados obtidos nas pessoas idosas com os resultados obtidos nos participantes mais novos, também com implante coclear.

Poissant e seus colaboradores, avaliaram a depressão e a solidão na QDV dos participantes e concluíram que o implante coclear melhora a QDV em termos da diminuição das pontuações encontradas nos resultados desse estudo, sendo que também não existem diferenças significativas entre os resultados obtidos pelos pacientes idosos ( $\geq 70$ anos) e os mais novos ( $\leq 60$ anos) [22]. Estes resultados, vão ao encontro das premissas da OMS no que respeita ao conceito de QDV na medida em que esta é definida como a perceção que o indivíduo tem sobre a sua posição na vida, dentro do contexto dos sistemas de cultura e valores nos quais está inserido em relação aos seus objetivos, expectativas, padrões e preocupações [36].

Nos estudos de Chung et al. [17], Orabi et al. [20] e Park et al. [21] os participantes idosos tinham idade igual ou superior a 65 anos. Orabi et al. [20] e Park et al. [21] referem que após o implante coclear os pacientes idosos têm melhorias na QDV. No entanto, Orabi et al. [20] não fazem a comparação com resultados obtidos por um grupo de pacientes mais novos. Chung et al. [17] obtiveram melhorias nas pontuações pós-implante coclear no grupo de pessoas idosas, contudo, os grupos de pacientes com menos de 65 anos conseguiram apresentar melhores resultados dado que apresentaram benefícios significativos num maior número de subescalas. O estudo de Eshraghi et al. [18] é o único que analisa a QDV num grupo de participantes com mais idade ( $\geq 79$ anos) o qual utiliza uma metodologia um pouco diferente de todos os outros autores pois estudaram a QDV em pessoas com implante coclear ligado ou desligado. Contudo, chegaram à conclusão que os seus participantes obtêm benefícios significativos com o implante coclear ligado, ao nível da QDV, pois quando estavam com o implante coclear desligado o grau de dificuldades era maior, tendo sido encontrada uma diferença significativa entre o antes (implante coclear desligado) e o após (implante coclear ligado) $(p<$ $0,001)$.

As diferenças encontradas nos estudos quanto à definição dos conceitos de pessoa idosa e intervalo de tempo
16

Port J Public Health 2017;35:10-18 DOI: $10.1159 / 000477645$
Brandão/Rebelo 
considerado para a avaliação dos pacientes após a implantação contribuem para uma dificuldade na interpretação dos resultados, tornando-se por isso difícil a comparação dos resultados obtidos nos diferentes estudos. A existência de diferentes amostras em cada estudo, algumas delas reduzidas, criou também dificuldades na obtenção de similaridades nos resultados obtidos. Estas dificuldades também foram encontradas por outros investigadores aquando da análise de estudos numa outra revisão sistemática [37].

Uma vez que a DA é uma patologia em que as pessoas não correm risco de vida, questiona-se se a colocação do implante coclear não será um procedimento demasiado dispendioso, e por isso dispensável, quando destinado a pessoas idosas, que vivem das suas reformas e supostamente com uma produtividade diminuída [11].

Contudo, apesar dessas pessoas poderem já não estar inseridas no mercado de trabalho, continuam a necessitar de ter uma boa QDV, o que apenas é possível quando conseguem comunicar com os seus pares de uma forma eficaz.

O implante coclear pode trazer muitos benefícios a esse nível, ajudando as pessoas a poderem mais facilmente interagir e comunicar com os outros, pelo que a sua vida social se torna mais positiva, e consequentemente as pessoas idosas melhoram a sua QDV como mostra o mais recente estudo realizado em pessoas implantadas com 80 anos ou mais [38].

Num atual contexto de crise económica, em que os gastos em saúde também são afetados, torna-se muito importante comprovar os benefícios que advêm da colocação do implante coclear nos idosos. Alguns estudos realizados nos EUA e na Europa demonstram que a implantação coclear tem uma relação custo/benefício favorável, desde que se comparem os custos da operação e do equipamento (que é dispendioso) com os custos que teriam de ser despendidos nos cuidados médicos, educação e apoio social ao longo da vida dos indivíduos com DA [39].

Outros estudos mostram ainda que os adultos reabilitados que estão em condições de voltarem ao mercado de trabalho, em resultado do implante coclear, irão representar menos gastos para o estado, dado que a probabilidade de necessitarem de apoios sociais será menor [40]. Num estudo realizado no Reino Unido, o custo-benefício do implante coclear em pessoas idosas, foi considerado mediano em relação aos portadores de próteses auditivas convencionais apenas devido à diferença na longevidade em relação aos mais novos [41]. Por outro lado, o implante coclear traz muitos benefícios ao nível da comunicação, ajudando as pessoas a poderem mais facilmente interagir

Instrumentos sobre QDV em Pessoas

Idosas após Implante Coclear com os outros, pelo que a sua vida social se torna mais positiva, e consequentemente as pessoas idosas melhoram a sua QDV como mostra o mais recente estudo realizado em pessoas implantadas com 80 anos ou mais [38]. No seguimento destas observações e numa era em que a esperança média de vida é cada vez mais elevada, pode-se questionar se não será mesmo importante a adoção do implante coclear mesmo nas pessoas com 65 ou mais anos de idade, público-alvo desta revisão.

$\mathrm{Na}$ avaliação da qualidade dos trabalhos, nenhum estudo correspondeu plenamente aos critérios para ser enquadrado na categoria A da classificação STROBE, porém todos mereceram enquadrar-se na categoria $B$, o que valoriza a credibilidade dos resultados

\section{Limitações e Perspetivas de Pesquisa}

No presente trabalho, a principal limitação diz respeito à falta de apresentação das características psicométricas da maioria dos instrumentos avaliados. Essa limitação, no entanto, não teve repercussão para o objetivo do presente estudo, pois os instrumentos foram analisados de modo a permitir mostrar quais são os utilizados em indivíduos com DA e principalmente qual é o instrumento específico (o único onde se incluem as características psicométricas), que mede a QDV em pessoas idosas submetidas a implantes cocleares. No entanto, é recomendável a pesquisa das características psicométricas de todos os instrumentos a avaliar num trabalho futuro.

\section{Conclusão}

Apenas um dos sete estudos selecionados contemplou um instrumento de avaliação específico, especialmente desenhado para avaliar a QDV das pessoas com implante coclear (NCIQ), incluindo as pessoas idosas.

$\mathrm{O}$ implante coclear mostrou ser efetivo em todos os grupos de participantes acima dos 65 anos de idade, pelo que deve ser considerado na população idosa com DA pós-lingual, sensorioneural, severa a profunda. $\mathrm{O}$ implante coclear pode trazer muitos benefícios para esta população, podendo ser um auxiliar de excelência na sua interação e comunicação com os pares, contribuindo para a sua participação e inclusão na vida social e, consequentemente, na melhoria da sua saúde e QDV.

Mais estudos são necessários para investigar a relevância do uso de instrumentos específicos, pois o uso de instrumentos genéricos pode criar limitações [17] à avaliação da QDV das pessoas após a colocação do implante coclear, incluindo as idosas. 


\section{References}

1 World Health Organization: International Day for Ear and Hearing. Geneva, WHO, 2012.

2 Bogardus ST, Yueh B, Shekelle PG: Screening and management of adult hearing loss in primary care: clinical applications. JAMA 2003 289:1986-1990.

3 Scarinci N, Worrall L, Hickson L: The ICF and third-party disability: its application to spouses of older people with hearing impairment. Disabil Rehabil 2009;31:2088-2100.

4 Harrison RV: Noise-induced hearing loss in children: a 'less than silent' environmental danger. J Paediatr Child Health 2008;13:377382.

5 Ciorba A, Bianchini C, Pelucchi S, Pastore A: The impact of hearing loss on the quality of life of elderly adults. Clin Interv Aging 2012; 7:159-163.

6 Almeida SP, Falcão JM: Incapacidade auditiva autodeclarada na população portuguesa: uma análise aos dados do quarto Inquérito Nacional de Saúde. Acta Med Port 2009;22:223232.

7 Gates GA, Mills JH: Presbycusis. Lancet 2005; 366:1111-1120.

8 Dalton DS, Cruickshanks KJ, Klein BE, Klein R, Wiley TL, Nondahl DM: The impact of hearing loss on quality of life in older adults. Gerontologist 2003;43:661-668.

9 Heine C, Browning CJ: Communication and psychosocial consequences of sensory loss in older adults: overview and rehabilitation directions. Disabil Rehabil 2002;24:763-773.

10 Huang Q, Tang J: Age-related hearing loss or presbycusis. Eur Arch Otorhinolaryngol 2010;267:1179-1191.

11 Andrade S, Peixoto C, Alves M, Martins JH, Veríssimo MT, Quadros J, et al: Reabilitação auditiva por implante coclear na população geriátrica. Cad Otorrinolaringol 2012;18:1-6

12 Gaylor JM, Raman G, Chung M, Lee J, Rao M, Lau J, et al: Cochlear implantation in adults: a systematic review and meta-analysis. JAMA Otolaryngol Head Neck Surg 2013;139:265272.

13 Hinderink JB, Krabbe PF, Van Den Broek P: Development and application of a health-related quality-of-life instrument for adults with cochlear implants: the Nijmegen cochlear implant questionnaire. Otolaryngol Head Neck Surg 2000;123:756-765.

14 Vandenbroucke JP, von Elm E, Altman DG, Gøtzsche PC, Mulrow CD, Pocock SJ, et al: Strengthening the Reporting of Observational Studies in Epidemiology (STROBE): explanation and elaboration. PLoS Med 2007;4:e297.
15 von Elm E, Altman DG, Egger M, Pocock SJ., Gøtzsche PC, Vandenbroucke JP, et al: The Strengthening the Reporting of Observational Studies in Epidemiology (STROBE) Statement: guidelines for reporting observational studies. PLoS Med 2007;4:e296.

16 Olmos M, Antelo M, Vazquez H, Smecuol E, Maurino E, Bai JC: Systematic review and meta-analysis of observational studies on the prevalence of fractures in coeliac disease. Dig Liver Dis 2008;40:46-53.

17 Chung J, Chueng K, Shipp D, Friesen L, Chen JM, Nedzelski JM, et al: Unilateral multichannel cochlear implantation results in significant improvement in quality of life. Otol Neurotol 2012;33:566-571.

18 Eshraghi AA, Rodriguez M, Balkany TJ, Telischi FF, Angeli S, Hodges AV, at al: Cochlear implant surgery in patients more than seventy-nine years old. Laryngoscope 2009;119: 1180-1183.

19 Olze H, Grabel S, Forster U, Zirke N, Huhnd LE, Haupt H, et al: Elderly patients benefit from cochlear implantation regarding auditory rehabilitation, quality of life, tinnitus, and stress. Laryngoscope 2012;122:196-203.

20 Orabi AA, Mawman D, Al-Zoubi F, Saeed SR, Ramsden RT: Cochlear implant outcomes and quality of life in the elderly: Manchester experience over 13 years. Clin Otolaryngol 2006;31:116-122.

21 Park E, Shipp DB, Chen JM, Nedzelski JM, Lin VY: Postlingually deaf adults of all ages derive equal benefits from unilateral multichannel cochlear implant. J Am Acad Audiol 2011;22:637-643.

22 Poissant SF, Beaudoin F, Huang J, Brodsky J, Lee DJ: Impact of cochlear implantation on speech understanding, depression, and loneliness in the elderly. Otolaryngol Head Neck Surg 2008;37:488-494.

23 Vermeire K, Brokx JP, Wuyts FL, Cochet E, Hofkens A, Van de Heyning PH: Quality-oflife benefit from cochlear implantation in the elderly. Otol Neurotol 2005;26:188-195

24 Robinson K, Gatehouse S, Browning GG: Measuring patient benefit from otorhinolaryngological surgery and therapy. Ann Otol Rhinol Laryngol 1996;105:415-422.

25 Ferreira PL: Criação da versão portuguesa do MOS SF-36. Parte I - Adaptação cultural e linguística. Acta Med Port 2000;13:55-66.

26 Ware JE, Sherbourne CD: The MOS 36-item short-form health survey (SF-36). I. Conceptual framework and item selection. Med Care 1992;30:473-483
27 Ferreira PL: Criação da versão portuguesa do MOS SF-36. Parte II - Testes de validação. Acta Med Port 2000b;13:119-127.

28 Yesavage JA, Brink TL, Rose TL, Lum O, Huang V, Adey M, et al: Development and validation of a geriatric depression screening scale: a preliminary report. J Psychiatr Res 1982; 17:37-49.

29 Chou R, Dana T, Bougatsos C, Fleming C, Beil $\mathrm{T}$ : Screening for Hearing Loss in Adults Ages 50 Years and Older: A Review of the Evidence for the U.S. Preventive Services Task Force. Rockville, Agency for Healthcare Research and Quality, 2011.

30 Russell DW: UCLA Loneliness Scale (Version 3): reliability, validity, and factor structure. J Pers Assess 1996;66:20-40.

31 Newton JP: Changes in the ageing process: a longer working life for some quality of life? Gerodontology 2006;23:193-194.

32 Ekwall AK, Sivberg B, Hallberg IR: Loneliness as a predictor of quality of life among older caregivers. J Adv Nurs 2005;49:23-32.

33 Ventry IM, Weinstein BE: The hearing handicap inventory for the elderly: a new tool. Ear Hear 1982;3:128-134.

34 Newman CW, Weinstein BE, Jacobson GP, Hug GA: The Hearing Handicap Inventory for Adults: psychometric adequacy and audiometric correlates. Ear Hear 1990;11:430433.

35 Buchman CA, Fucci MJ, Luxford WM: Cochlear implants in the geriatric population. Ear Nose Throat J 1999;78:489-494.

36 WHOQOL Group: Development of the WHOQOL: rationale and current status. Int J Ment Health Nurs 1994;23:24-56.

37 Yeagle JD, Ceh KM, Francis HW: Geriatric cochlear implantation. Oper Tech Otolaryngol Head Neck Surg 2010;21:266-271.

38 Cloutier F, Bussières R, Ferron P, Côté M OCTO "Outcomes of cochlear implant for the octogenarians: audiologic and quality-oflife". Otol Neurotol 2014;35:22-28.

39 Manrique M, Ramos A, Morera C, Cenjor C, Lavilla MJ, Boleas MS, et al: Analysis of the cochlear implant as a treatment technique for profound hearing loss in pre and postlocutive patients. Acta Otorrinolaringol Esp 2006;57: 2-23.

40 Carter R, Hailey D: Economic evaluation of the cochlear implant. Int J Technol Assess Health Care 1999; 15:520-530.

41 UK Cochlear Implant Study Group: Criteria of candidacy for unilateral cochlear implantation in postlingually deafened adults III: prospective evaluation of an actuarial approach to defining a criterion. Ear Hear 2004;25 361-374. 